\title{
Isolated Noncompaction of the Myocardium
}

\author{
Jorge Elias, W ilson Valadão, Ricardo Kuniyoshi, Aloir Q ueiroz, Carlos André Peixoto
}

Vitória, ES - Brazil

\begin{abstract}
We report the case of a 42-year-old female with fatigue on exertion and palpitation consequent to the existence of isolated noncompaction of the myocardium. We discuss clinical and familial findings, diagnostic possibilities, and prognostic and therapeutical implications of this rare disorder of endomyocardial morphogenesis.
\end{abstract}

During the initial phase of embryonic development, the heart is a trabecular net with a spongy myocardium. The intertrabecular spaces communicate with the cardiac chambers. As the heart develops, the myocardium condenses and the intertrabecular recesses are reduced to capillaries. Trabecular compaction is usually more complete in the left ventricle than in the right ventricle. Noncompaction of the ventricular myocardium is defined as an anomaly of endomyocardial morphogenesis, and it is believed to be an arrest in the compaction of the myocardial fibers, which meet forming an interwoven loose net during intrauterine life. Persistence of the noncompaction of the myocardium is a rare entity, usually diagnosed in the pediatric population and associated with other structural congenital malformations of the heart. It predominates in patients with congenital obstruction of the right or left ventricle outflow tract ${ }^{1-4}$. Isolated noncompaction of the myocardium, defined by absence of any other associated structural cardiac alteration, is an even rarer form of the entity, with only a few reports in the literature ${ }^{5-8}$. Isolated noncompaction of the myocardium may be identified from childhood to adulthood. Its diagnosis may be delayed because of some findings resembling other clinical entities. Both sexes may be affected and familial recurrence may occur.

We report the case of a 42-year-old female with isolated noncompaction of the myocardium, describing the clinical and familial history, diagnostic possibilities, and therapeutical approach. We also review the literature about this rare disorder of endomyocardial morphogenesis.

\section{Case report}

A 42-year-old female complaining of fatigue on medi-

Instituto de Cardiologia do Espírito Santo - Clínica do Ritmo

Mailing address: Jorge Elias - Rua Alaor Queiroz de Araujo, 220 - 29055-010 Vitória, ES, Brazil um exertion, chest pain, and palpitation for 10 years was referred to our service to investigate arrhythmia. She reported loosing 2 children in the first year of life, one due to sudden death at the age of 5 months and the other due to refractory heart failure in the first week of life. The anatomicopathological study of the latter child performed in another service showed a dilated left ventricle with increased and abnormal trabeculation, whose final diagnosis, at the time, was cardiomyopathy of undetermined etiology. She also reported two siblings who had sudden death during childhood, and one uncle and one cousin, who died due to refractory congestive heart failure. The physical examination was normal. The 12-lead electrocardiogram showed sinus rhythm and deviation of the axis to the right in the frontal plane without meeting the criteria of left posteroinferior divisional heart block (figura 1A). Chest X-ray was normal. The exercise test showed depression of the ST segment associated with typical precordialgia (figura 1C). Technetium-sestamibi myocardial scintigraphy showed a fixed area of reduced uptake in the anteroapical projection. Coronary angiography showed normal coronary arteries and a left ventricle with a trabecular and hypertrophic pattern predominating in the apical region (figura 2). Holter monitoring showed a frequent, symptomatic, monomorphic ventricular ectopia with the morphology of left bundle-branch block in V1 (figura 1B).

Diagnosis of isolated noncompaction of the myocardium was based on the following findings on Doppler echocardiography: 1) absence of another coexisting heart anomaly; 2) alterations in the myocardial structure characterized by excessive and prominent trabeculation mainly in the apical region; 3 ) intertrabecular spaces perfused by the ventricular cavity visualized on color Doppler (figure 3). The echocardiogram also showed anteroseptal akinesia. The ejection fraction calculated on the radionuclide ventriculography was $49 \%$. High-resolution electrocardiogram showed absence of late ventricular potentials. Electrophysiological study performed with no drugs did not show sustained ventricular tachycardia inducible with a protocol of up to 3 extrastimuli at the level of the right ventricular apex and outflow tract.

An extensive familial investigation was carried out with electrocardiography and two-dimensional echocardiography. No other individuals meeting the criteria of isolated noncompaction of the myocardium were identified. Initially, the patient was kept without medication for a period of 2 years. Because of palpitation, precordialgia, and the risk of thromboembolic phenomena attributed to this disorder, 


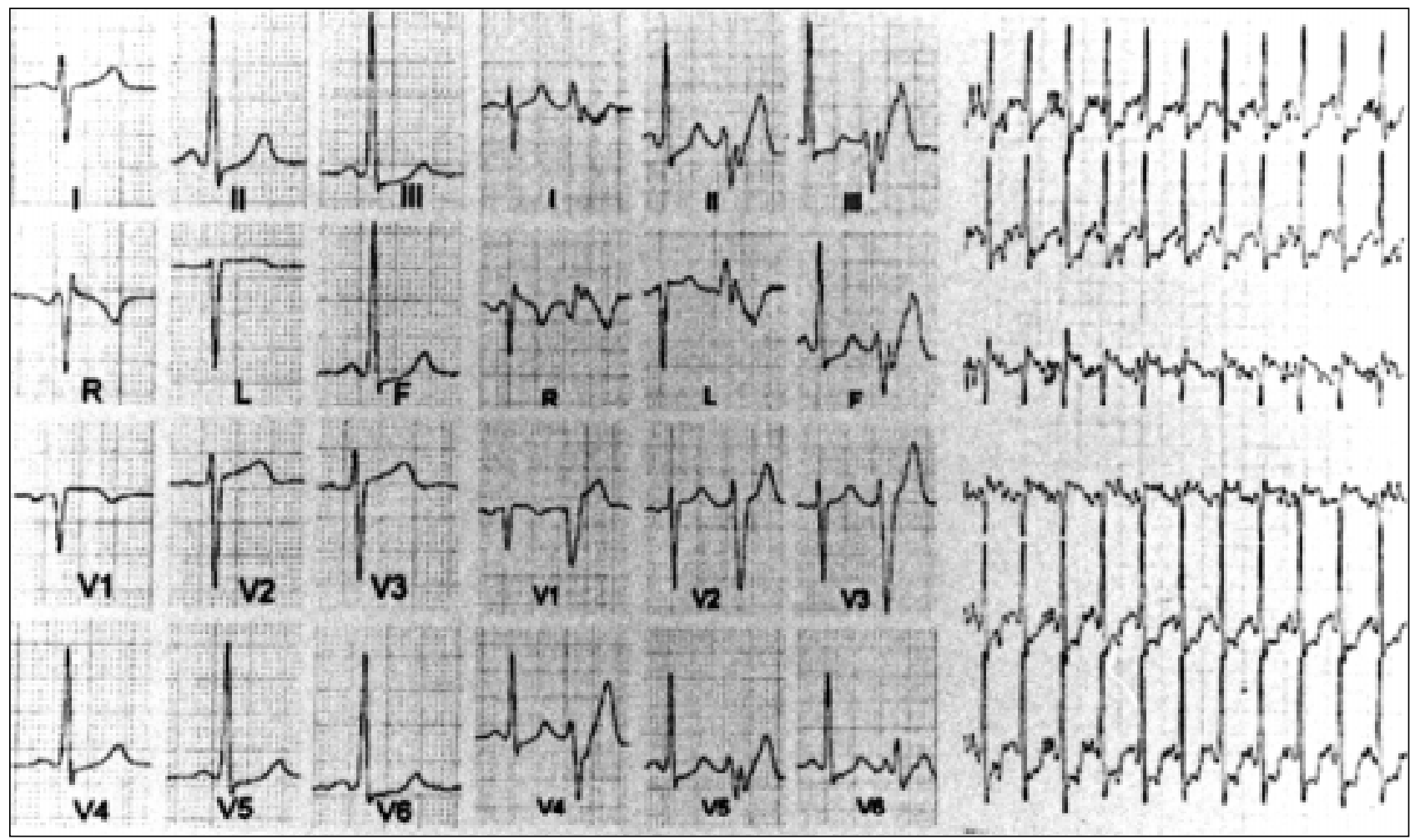

Fig. 1 -A) Twelve-lead electrocardiogram showing sinus rhythm and deviation of the axis to the right in the frontal plane; B) ventricular ectopia with left bundle-branch block morphology in the horizontal plane; C) depression of the ST segment with a rectified pattern and association with complaint of precordialgia during the exercise test.
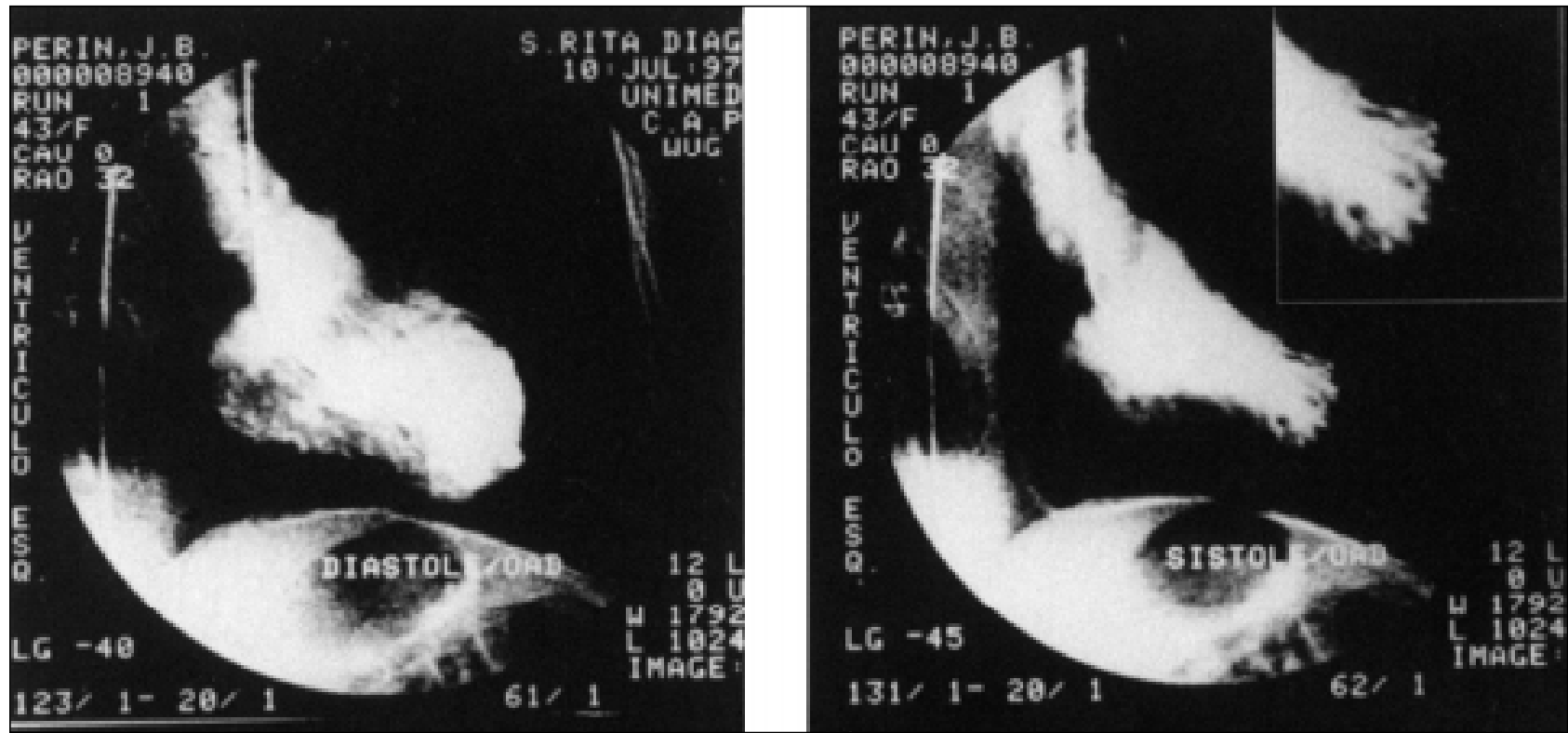

Fig. 2 - Ventriculography in right anterior oblique view during diastole and systole showing an increase in trabeculation in the apical region.

therapeutics with atenolol and oral anticoagulants was introduced. In a 3-year ambulatory follow-up, the patient has remained stable and asymptomatic.

\section{Discussion}

Noncompaction of the myocardium is characterized by numerous and prominent trabeculae with intertrabecular recesses that deeply penetrate the left ventricular myocardium. In the majority of the reports in the literature, noncompaction of the ventricular myocardium is associated with other congenital heart diseases, among which pulmonary atresia and obstruction of the left ventricular outflow tract combined with an intact interventricular septum predominate ${ }^{1-3}$. In these 


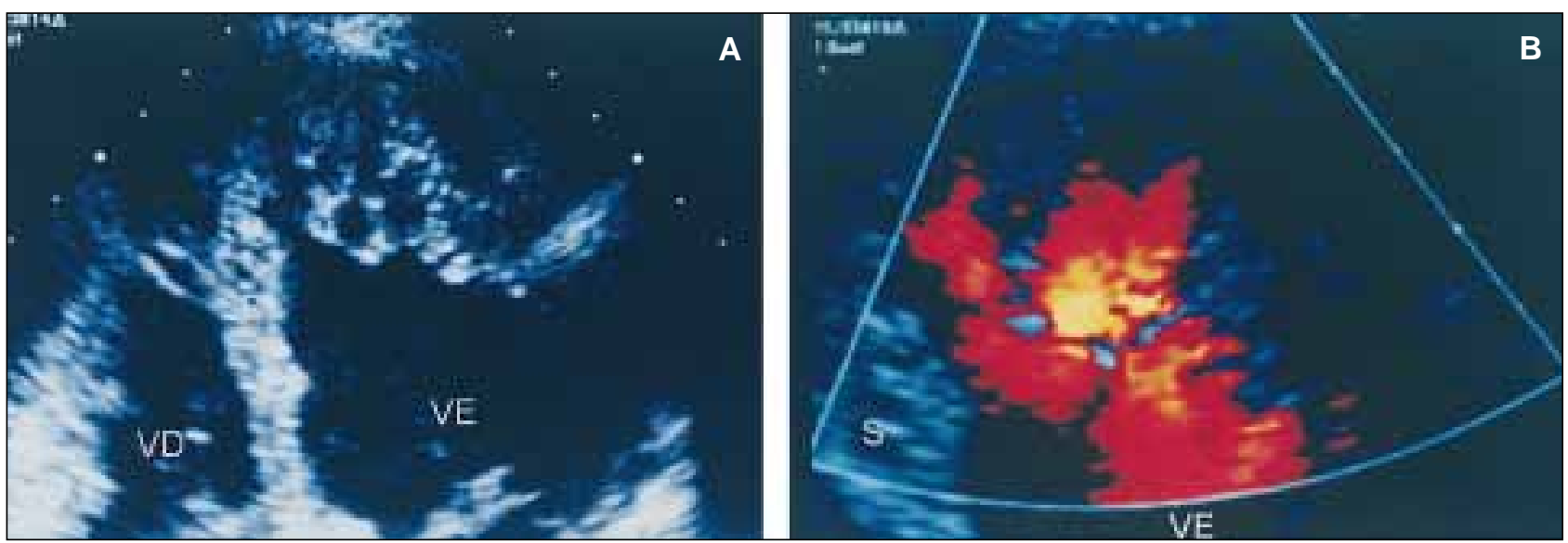

Fig. 3-A) Echocardiogram in apical four-chamber view showing prominent trabeculation in the left ventricular apical region B) with perfused intertrabecular recesses evidenced on color Doppler.

cases, noncompaction of the ventricular myocardium occurs because the ventricles are exposed to very high pressures during the intrauterine development. Noncompaction of the myocardium has also been identified associated with abnormalities of the origin of the left coronary artery from the trunk of the pulmonary artery ${ }^{4}$. In regard to the isolated noncompaction of the myocardium, its cause is still unknown and, so far, no factor that could justify an arrest in the ventricular myocardium compaction has been identified. Chin et $\mathrm{l}^{5}$ carried out histopathological studies in individuals with isolated noncompaction of the myocardium, and they showed an excessive trabeculation, mainly in the apical region of the heart, associated with continuity between the left ventricular myocardium and the deep intertrabecular recesses. They concluded that these findings expressed an arrest in the compaction of the ventricular myocardium, therefore justifying that the term noncompaction is more appropriate than the term persistence of the ventricular sinusoids. These findings were confirmed by Ritter et $\mathrm{al}^{6}{ }^{6}$ in a recent publication that gathered the largest case series of this entity. In this latter study, similarly to the investigation conducted by Chin et $\mathrm{al}^{5}$, isolated noncompaction of the myocardium was mainly located in the apical region of the heart. However, isolated noncompaction of the myocardium was evidenced in both ventricles, showing a variation in the presentation of the disorder. In both studies, the anatomicopathological findings and the location of the isolated noncompaction of the myocardium were in accordance with the echocardiographic findings. Based on this correlation between the anatomicopathological and the echocardiographic findings, Chin et al ${ }^{5}$ proposed that the identification of structural changes on echocardiogram would be enough to confirm the diagnosis of isolated noncompaction of the myocardium. In this study, the authors suggested that the diagnosis of isolated noncompaction of the myocardium would be based on the echocardiogram on the presence of numerous and prominent trabeculae and of deep intertrabecular recesses in the absence of coexisting heart anomalies. The authors observed that the trabeculae were fewer and less prominent close to the mitral valve than in the apex of the left ventricle With a left ventricular free wall thickness of $8.6 \pm 1.6 \mathrm{~m}$ mat the level of the mitral valve and $22.9 \pm 1.2 \mathrm{~mm}$ in the apex of the left ventricle. Ritter et al ${ }^{6}$ observed on color Doppler that the perfusion of the intertrabecular spaces showed a continuous flow leaving the ventricular cavity. In addition to the apical region, the anterior, lateral, and inferior regions may also show characteristic structural changes.

Our patient met all the proposed criteria for the diagnosis of isolated noncompaction of the myocardium ${ }^{5}$. In the study by Ritter et al ${ }^{6}$, the prevalence of isolated noncompaction of the myocardium on the echocardiogram was $0.05 \%$ of the cases studied; isolated impairment of the left ventricle occurred in 59\% of the patients and biventricular impairment occurred in $41 \%$ of the patients. Computed tomography with contrast medium may be a complementary test for diagnostic confirmation enabling the differentiation between compacted and noncompacted tissues ${ }^{7}$.

The most common findings on the electrocardiogram in sinus rhythm are as follows: presence of incomplete and complete left bundle-branch block, disorders of intraventricular conduction, changes in the ventricular repolarization, but in some cases, the electrocardiogram is normal ${ }^{8}$.

In our case, an interesting finding was the presence of electrocardiographic changes suggesting myocardial ischemia associated with typical precordialgia during the exercise test. In addition, on scintigraphic study, a reduction in the uptake of a fixed area in the anteroapical region was identified and, on the echocardiogram, a pattern of anteroseptal akinesia was observed. Even though we have not found similar clinical findings in the literature, we have considered that the presence of ischemia in this entity may be due to functional changes resulting from a regional increase in the demand of oxygen and a decrease in the reserve of coronary flow to the subendocardial region. On the histopathological examination of the isolated noncompaction of the myocardium, ischemic lesions in the prominent trabecular regions occurred ${ }^{6}$. The finding of myocardial ischemia in the isolated noncompaction of the myocardium and its prognostic implication require further investigation. Clinical findings may vary from asymptomatic patients to patients with progressive left ventricular dysfunction with arrhythmias and systemic and pulmonary embolic phenomena. Six years after diagnosis, $59 \%$ of the patients in the study by Ritter et $\mathrm{al}^{6}$ had died or had been 
referred for heart transplantation. Data from the literature show a better prognosis for the asymptomatic patients ${ }^{6}$. Even though the cause of the progressive depression of the left ventricular function in the isolated noncompaction of the myocardium has not yet been well clarified, clinical and echocardiographic findings resemble those of dilated cardiomyopathy, except that in the former disorder the left ventricular wall is thickened in the apical and inferior segments. In dilated cardiomyopathy, some degree of inferoapical trabeculation associated with the intertrabecular spaces may be visualized; therefore, a distinction between these 2 disorders, at least from the morphological point of view, is not always clear. In spite of the fact that demonstration on color Doppler echocardiography of deep perfused intertrabecular recesses is one of the markers for the diagnosis of isolated noncompaction of the myocardium, transitional variations between the isolated noncompaction of the myocardium and dilated cardiomyopathy may exist. In addition, more discreet cases of isolated noncompaction of the myocardium without diagnostic confirmation may exist, cases lacking excessive trabeculation in the inferoapical region, hypertrophy, and marked intertrabecular recesses. Ritter et al ${ }^{6}$, based on the finding that all patients with ventricular tachycardia detected by Holter monitoring have severe systolic dysfunction of the left ventricle, have suggested that an altered left ventricular performance may be one of the major causes of the high incidence of left ventricular arrhythmia in this entity. Increase in scaring and fibrosis in areas of excessive trabeculation and hypertrophy would be another major factor. The authors believe that isolated noncompaction of the myocardium cannot be compared with arrhythmogenic dysplasia of the right ventricle because, even though both diseases have an arrhythmogenic potential, the morphologic characteristics of the latter are totally different. The high incidence of thromboembolic phenomena in the isolated noncompaction of the myocardium could result in formation of local thrombi in the deep intertrabecular recesses in addition to ventricular dysfunction. Based on the high incidence of thromboembolic events in their case series, Ritter et al ${ }^{6}$ suggested that patients with isolated noncompaction of the myocardium confirmed on echocardiography should undergo systemic anticoagulation, independently from identification of thrombi visualized on echocardiography. The clinical treatment of patients with ventricular dysfunction should be the one usually used in patients with ventricular failure; heart transplantation should be considered in those cases refractory to the more conservative treatment. Incidence of ventricular failure, ventricular arrhythmia, and embolizations should draw attention to this rare entity, which is complicated by a considerable morbidity and high mortality.

Familial recurrence of isolated noncompaction of the myocardium was identified in cases associated with the Melnick-Needles syndrome, which comprises unusual bone modeling characterized by facial dysmorphism and occasionally multiple anomalies ${ }^{9}$. Recently, Bley et al ${ }^{10}$ identified, through the genetic analysis of linkage in a family with the Barth syndrome, the presence of Xq28-linked isolated noncompaction of the myocardium. In the study by Ritter et $\mathrm{al}^{6}, 2$ cases with familial antecedents but without any other associated malformations were identified.

Our patient had a newborn baby who died because of undetermined cardiomyopathy. Description of the autopsy suggested isolated noncompaction of the myocardium. It was not possible to investigate the other cases of death in childhood in the family of the patient; we can only formulate the hypothesis of isolated noncompaction of the myocardium. The investigation conducted in the first-degree relatives of our patient did not identify other individuals with echocardiographic criteria for isolated noncompaction of the myocardium.

In conclusion, the characteristic morphological findings of isolated noncompaction of the myocardium may be confirmed on the two-dimensional Doppler echocardiogram, and these findings correspond with the macroscopic morphologic findings at the autopsy. This diagnosis should be considered in patients with ventricular failure, ventricular arrhythmia, and embolic events. Familial investigation through echocardiography should be conducted in the relatives of a patient with the diagnosis of isolated noncompaction of the myocardium. Even though this disorder has a high mortality rate, our report suggests that cases with a good myocardial function and absence of arrhythmia demonstrated through normal specific investigative tests may have a good prognosis.

\section{References}

1. Bellet S, Gouley BA. Congenital heart disease with multiple cardiac anomalies: report of a case showing aortic atresia, fibrous scar in myocardium and embryonal sinusoidal remains. Am J Med Sci 1932; 183: 458-65.

2. Davignon AL, DuShane JW, Kincaid OW, Swan HJC. Pulmonary atresia with intact ventricular septum: report of two cases studied by selective angiocardiography and right heart catheterization. Am Heart J 1961; 62: 690-7.

3. Lauere RM, Fink HP, Petry EL, Dunn MI, Diehl AM. Angiographic demonstration of intramyocardial sinusoids in pulmonary-valve atresia with intact ventricular septum and hypoplastic right ventricle. N Engl J Med 1964; 271: 68-72.

4. Dusek J, Bohuslav O, Duskova M. Posnatal persistence of spongy myocardium with embryonic blood supply. Arch Pathol 1975; 99: 312-17.

5. Chin TK, PerloffJK, Williams RG, Jue K, Mohrmann R. Isolated noncom- paction of left ventricular myocardium. A study of eight cases. Circulation 1990; 82: 507-13.

6. Ritter M, Oechslin E, Sütsch G, et al. Isolated noncompaction of the myocardium in adults. Mayo Clin Proc 1997; 72: 26-31.

7. Dewey J, Conces Jr DJ, Ryan T, Tarver RD. Noncompaction of ventricular myocardium: CT appereance. Am J Roent 1991; 156: 717-8.

8. Robida A, Hajar HA. Ventricular conduction defect in isolated noncompaction of the ventricular myocardium. Pediatr Cardiol 1996; 17: 189-91.

9. Wong JA, Bofinger MK. Noncompaction of the ventricular myocardium in Mel nick-Needles. Am J Med Genet 1997; 71: 72-5.

10. Bley SB, Mumford BR, Brown-Harrison MC, et al. Xq28-linked noncompaction of the left ventricular myocardium: prenatal diagnosis and pathologic analysis of affected individuals. Am J Med Genet 1997; 72: 257-65. 